\title{
Synthesis of pyrimido[1,6-a]quinoxalines via intermolecular trapping of thermally generated acyl(quinoxalin-2-yl)ketenes by Schiff bases
}

\author{
Svetlana O. Kasatkina, Ekaterina E. Stepanova*, Maksim V. Dmitriev, Ivan G. Mokrushin \\ and Andrey N. Maslivets*
}

\section{Full Research Paper}

Address:

Department of Chemistry, Perm State University, ul. Bukireva 15,

Perm 614990, Russian Federation

Email:

Ekaterina E. Stepanova* - caterina.stepanova@psu.ru;

Andrey N. Maslivets* - koh2@psu.ru

* Corresponding author

Keywords:

acyl(quinoxalin-2-yl)ketenes; cycloaddition;

pyrimido[1,6-a]quinoxalines; Schiff bases; thermolysis

\author{
Beilstein J. Org. Chem. 2018, 14, 1734-1742. \\ doi:10.3762/bjoc.14.147 \\ Received: 06 April 2018 \\ Accepted: 26 June 2018 \\ Published: 11 July 2018 \\ Associate Editor: T. J. J. Müller \\ (C) 2018 Kasatkina et al.; licensee Beilstein-Institut. \\ License and terms: see end of document.
}

\begin{abstract}
Acyl(quinoxalin-2-yl)ketenes generated by thermal decarbonylation of 3-acylpyrrolo[1,2-a]quinoxaline-1,2,4(5H)-triones react regioselectively with Schiff bases under solvent-free conditions to form pyrimido[1,6-a]quinoxaline derivatives in good yields.
\end{abstract}

\section{Introduction}

Quinoxaline is a 4-aza isostere of quinoline, which rarely occurs in structures of natural products. Its derivatives are gaining popularity in medicinal chemistry and pharmacology because many of them exhibit various biological activities $[1,2]$.

Quinoxaline-based 6/6/6-angularly fused scaffolds (quinoxaline fused by a six-membered heterocycle at the $[a]$-side) are promising biologically active compounds. Recent research studies revealed that they can act as inhibitors of poly(ADPribose) polymerase (PARP) [3], inhibitors of hepatitis $\mathrm{C}$ virus [4], 5- $\mathrm{HT}_{2 \mathrm{C}}$ agonists [5-7], substances for controlling intraocular pressure (IOP) [8] etc. (Figure 1).
Pyrimido[1,6- $a]$ quinoxalines are one of the most intriguing and unexplored structures representing isosteres of this scaffold. Only few synthetic procedures towards these compounds are described in the literature: heterocyclizations of $\alpha$-chloroisocyanates with quinoxalin-2-ylideneacetates [9], multicomponent Mannich-Ritter transformations of quinoxalin-2(1H)-ones under the action of nitriles and 3,4-dihydro-2H-pyran [10] and a microwave-assisted cascade strategy via in situ-generated $\mathrm{N}$-acyliminium ion precursors and amines [11] (Figure 2).

To develop a new synthetic approach towards pyrimido[1,6- $a$ quinoxalines we looked through the procedures to their closest 
<smiles>O=C1Nc2cc(Cl)c(Cl)cc2N2CCNC[C@@H]12</smiles>

full $5-\mathrm{HT}_{2 \mathrm{C}}$ agonist (WAY-161503); substance for controlling IOP
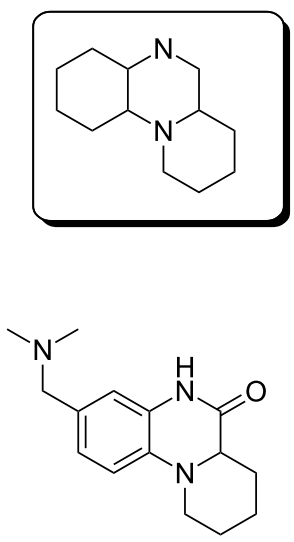

inhibitor of PARP

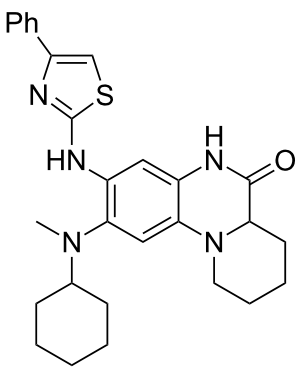

inhibitor of hepatitis $C$ virus

Figure 1: Quinoxaline-based 6/6/6-angularly fused scaffolds and respective examples of biologically active compounds.
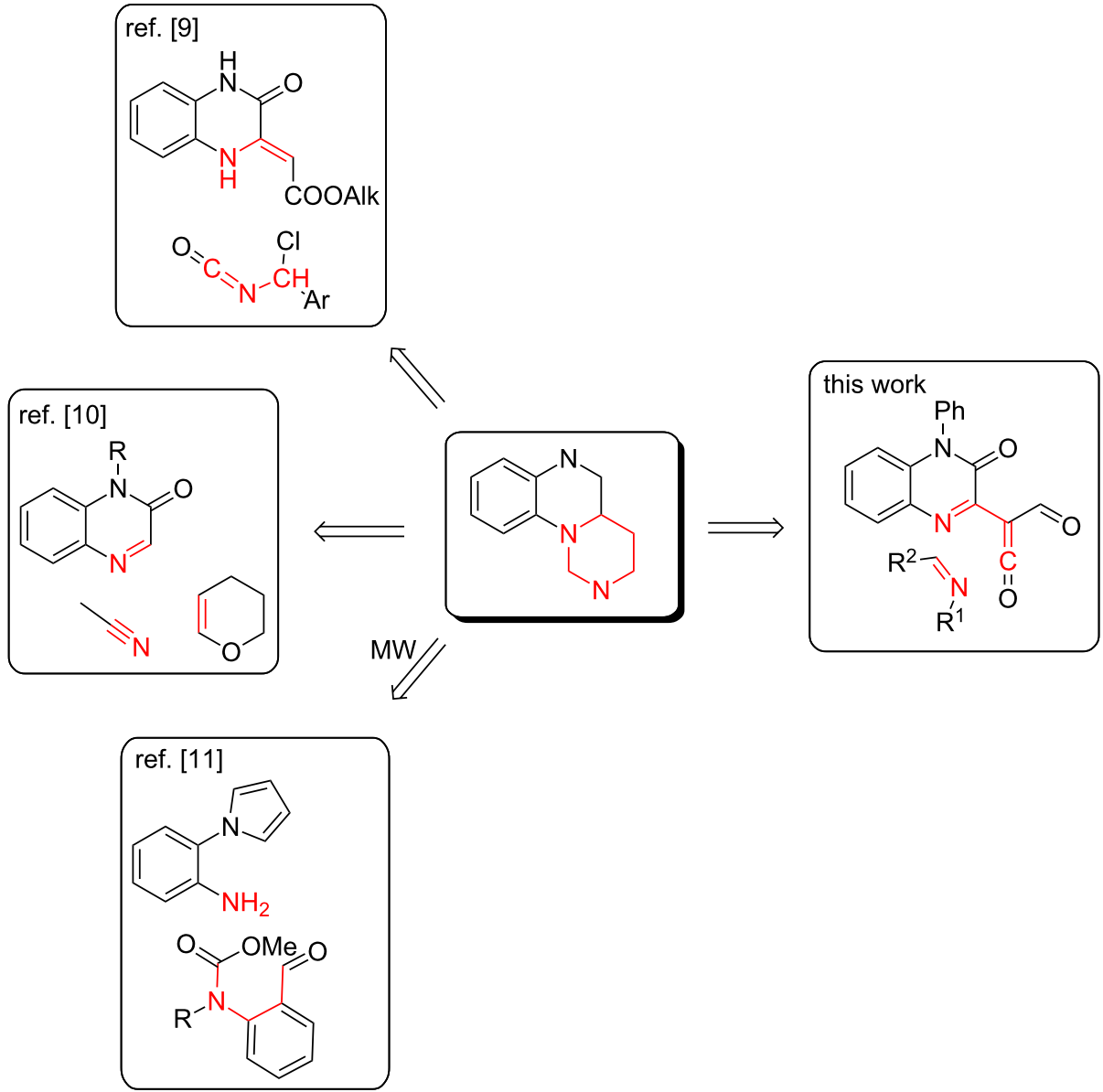

Figure 2: Synthetic routes towards the pyrimido[1,6-a]quinoxaline scaffold. 
analogues - pyrido[1,2- $a$ ]quinoxalines, the synthesis of which has been explored more frequently [3,4,12-46]. The analysis helped us to disclose a tempting but challenging methodology, which has the potential to be extended for the synthesis of the desired heterocyclic system, via intermolecular trapping of thermally generated acyl(quinoxalin-2-yl)ketenes [20,21,23,24,28,29,38] (Figure 2).

Syntheses utilizing acylketenes are of practical and theoretical interest due to the high reactivity of acylketenes and the structural diversity of the reaction products [47-54]. The introduction of the quinoxalin-2-yl substituent into acylketenes results in the formation of a peculiar system of conjugated double bonds, which can potentially act as either oxo-diene or aza-diene (Figure 3).<smiles>O=C=C(C=O)c1cnc2ccccc2n1</smiles>

Figure 3: Acyl(quinoxalin-2-yl)ketene.

To the best of our knowledge, there is no example of the involvement of the aza-diene fragment of acyl(quinoxalin-2yl)ketenes into intermolecular trapping by hetero-dienophiles published so far. In this article we report a synthetic protocol towards pyrimido[1,6- $a$ ]quinoxalines via the intermolecular trapping of acyl(quinoxalin-2-yl)ketenes by Schiff bases.

\section{Results and Discussion}

The most convenient method for the generation of acyl(quinoxalin-2-yl)ketenes is the thermal decarbonylation (thermolysis) of five-membered 2,3-dioxoheterocycles having a quinoxaline fragment. Currently, three types of such precursors are known: 5-aryl-4-quinoxalin-2-ylfuran-2,3-diones I [21], 3-aroyl-4arylpyrrolo[1,2-a]quinoxaline-1,2-diones II [55], and 3-acylpyrrolo[1,2-a]quinoxaline-1,2,4(5H)-triones III [23,56] (Scheme 1).

According to the literature data, precursors I and II are unsuitable for achieving the proposed goal as the generated ketene IV reacts only at its oxo-diene fragment in intermolecular trapping reactions with various dienophiles [57-62]. Under these circumstances precursors III generating ketenes $\mathbf{V}$ seemed to be the only suitable candidates for the development of a strategy towards pyrimido[1,6- $a]$ quinoxalines.

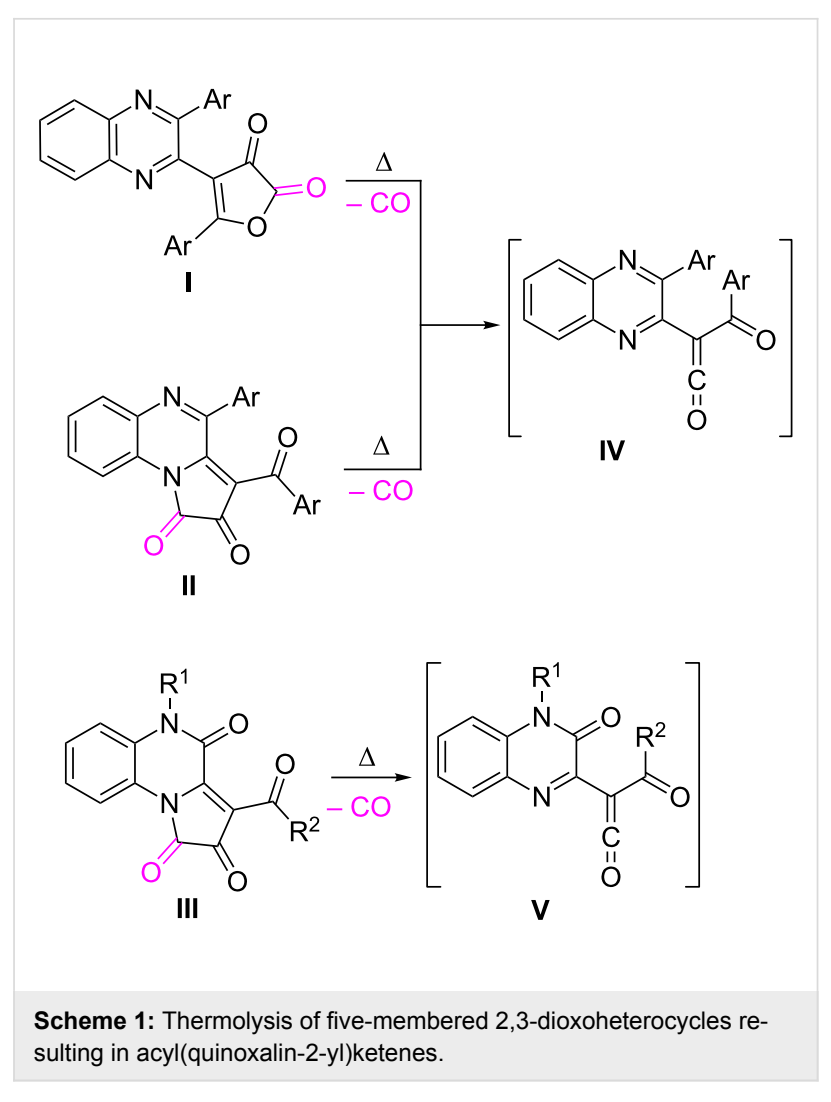

First, we studied the decarbonylation of precursors III 3-acylpyrrolo[1,2-a]quinoxaline-1,2,4(5H)-triones (PQTs, $\mathbf{1 a}-\mathbf{h}$ ) by simultaneous thermal analysis (STA, Table 1). According to the data obtained, PQTs 1a-h underwent thermal decomposition with a mass loss accompanied by an endothermic effect and $\mathrm{CO}$ evolution (Figure 4). The values of the mass loss corresponded to the elimination of a CO molecule from a PQT.

Table 1: Thermal characteristics of decarbonylation of PQTs 1a-h.

\begin{tabular}{cccc} 
PQT & \multicolumn{3}{c}{ temp. of decarbonylation $\left({ }^{\circ} \mathrm{C}\right)$} \\
\cline { 2 - 4 } & onset & extrapolated onset & peak \\
\hline 1a & 187 & 207 & 216 \\
1b & 174 & 209 & 220 \\
1c & 173 & 183 & 206 \\
1d & 148 & 174 & 183 \\
1e & 172 & 204 & 217 \\
1f & 179 & 198 & 212 \\
1g & 184 & 203 & 213 \\
1h & 171 & 197 & 206
\end{tabular}

Having taken into account the results of the thermal analysis, we examined the feasibility and conditions of the intermolecular reaction of the ketene generated from PQT 1a with benzal- 


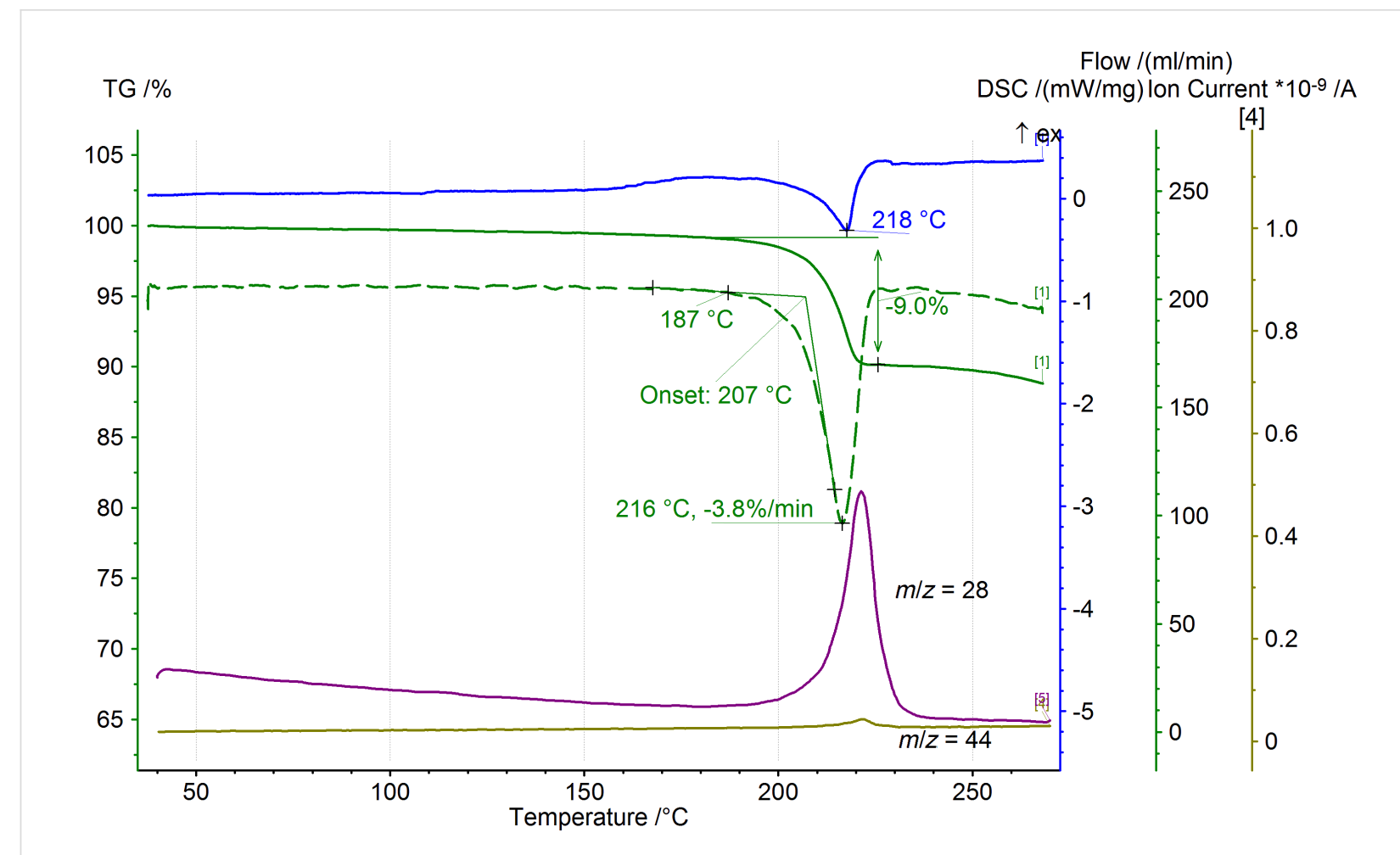

Figure 4: STA plot of thermolysis of PQT 1a. Blue solid curve: DSC; green solid curve: TG; greed dashed curve: DTG; violet solid curve: $\operatorname{MID}(\mathrm{m} / \mathrm{z}=28)$; brown solid curve: $\operatorname{MID}(\mathrm{m} / \mathrm{z}=44)$; heating rate: $5^{\circ} \mathrm{C} / \mathrm{min}$.

aniline (2a). The reaction mixtures obtained were investigated by UPLC-MS and the results are summarized in Table 2 .

The reaction mixtures contained only three types of products, and we succeeded to identify each of them. The structures of the reaction products were elucidated as the desired pyrimido[1,6a] quinoxaline 3a, quinoxalinone 4a [29] and pyrido[1,2a] quinoxaline 5a [29] (Scheme 2). Product IV of an alternative intermolecular trapping reaction (Table 1) was not detected.

The most likely way of the formation of quinoxalinone $\mathbf{4 a}$ is hydration of the ketene with subsequent decarboxylation (Scheme 2); more careful drying the reaction vials and solvents easily reduced the amount of compound $\mathbf{4 a}$.

The formation of pyrido[1,2- $a$ ]quinoxaline 5a can be explained by a concurrent process of ketene dimerization (Scheme 2) [29] in comparison to the intermolecular trapping of it by benzalaniline (2a). Since the yields of the target product 3a decreased and the yields of compound $\mathbf{5 a}$ increased at prolonged time of reaction, the formation of the target compounds deemed to be reversible.

Performing the reaction under solvent-free conditions at the onset decarbonylation temperature (Table 1) exceeded our expectations and gave the best yields of the target compound 3a (Table 2, entry 6).

Being inspired by the optimization results obtained, we examined the scope of the reaction applying the developed methodology with PQTs $\mathbf{1 a}-\mathbf{h}$ and Schiff bases $\mathbf{2 a}-\mathbf{d}$. The results are shown in Figure 5.

Unfortunately, our attempts to involve Schiff bases synthesized from aliphatic aldehydes and ketones did not give any satisfactory results because of various nucleophilic side-reactions.

We found that the intermolecular trapping worked perfectly in case of $\mathrm{N}^{5}$-substituted PQTs $1 \mathbf{a}-\mathbf{f}$ and did not work at all with $\mathrm{N}^{5}$-unsubstituted PQT $\mathbf{1 g}$ and $\mathbf{1 h}$. The failure to obtain products $\mathbf{3 o}$ and $\mathbf{3 p}$ from PQTs $\mathbf{1 g}$ and $\mathbf{1 h}$ can be explained by the occurrence of intramolecular cyclization in these ketenes resulting in the formation of furoquinoxalines $\mathbf{6 a}, \mathbf{b}[56,63,64]$ which were confirmed by UPLC-MS data as the sole products of the reaction (Scheme 3).

The formation of pyrimido[1,6-a]quinoxalines 3a-n was unambiguously confirmed by the crystal structure of compounds 3g and 3j (CCDC 1834011, Figure 6; CCDC 1834012, Figure 7). 
Table 2: Intermolecular trapping of ketene generated from PQT 1a by benzalaniline (2a) ${ }^{\mathrm{a}}$.

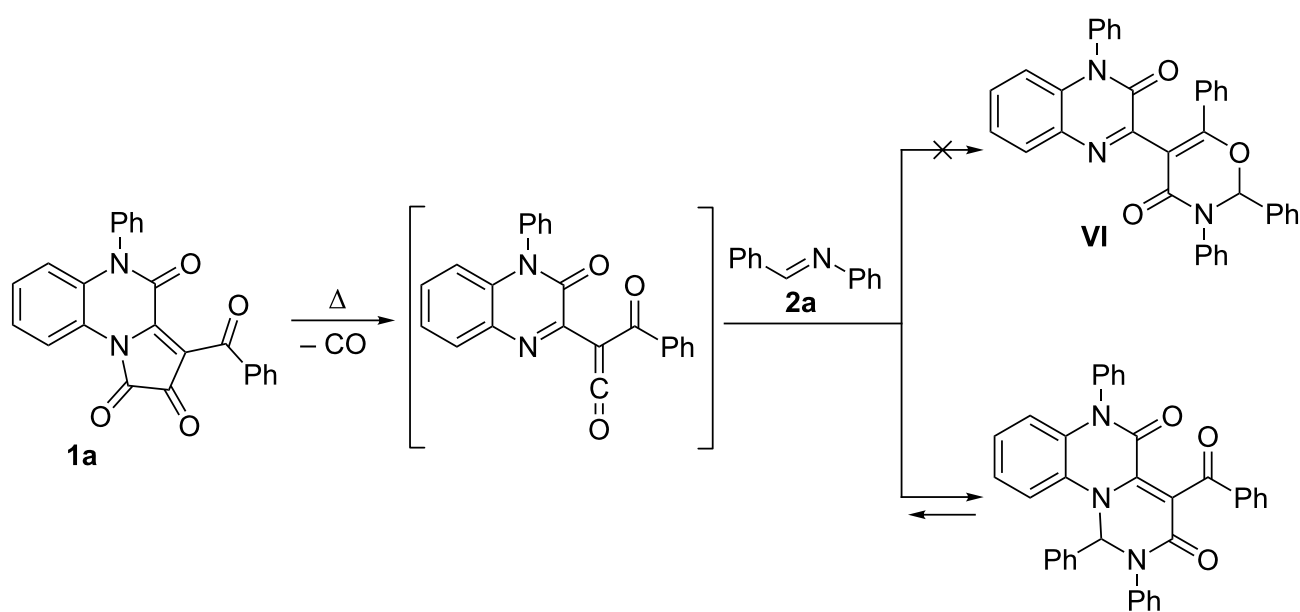

$3 \mathbf{a}$

\begin{tabular}{llll}
\hline entry & yield of $3 a(\%)^{\mathrm{b}}$ & time $(\mathrm{min})$ & temp. $\left({ }^{\circ} \mathrm{C}\right)$ \\
\hline 1 & 65 & 5 & 190 \\
2 & 50 & 15 & 190 \\
3 & 57 & 5 & 200 \\
4 & 30 & 60 & 200 \\
5 & 80 & 3 & 175 \\
6 & $85^{\mathrm{c}}$ & 2 & 187 \\
\hline
\end{tabular}

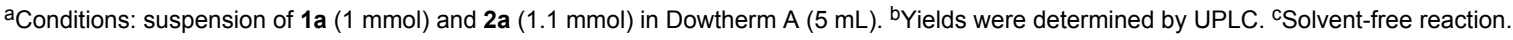

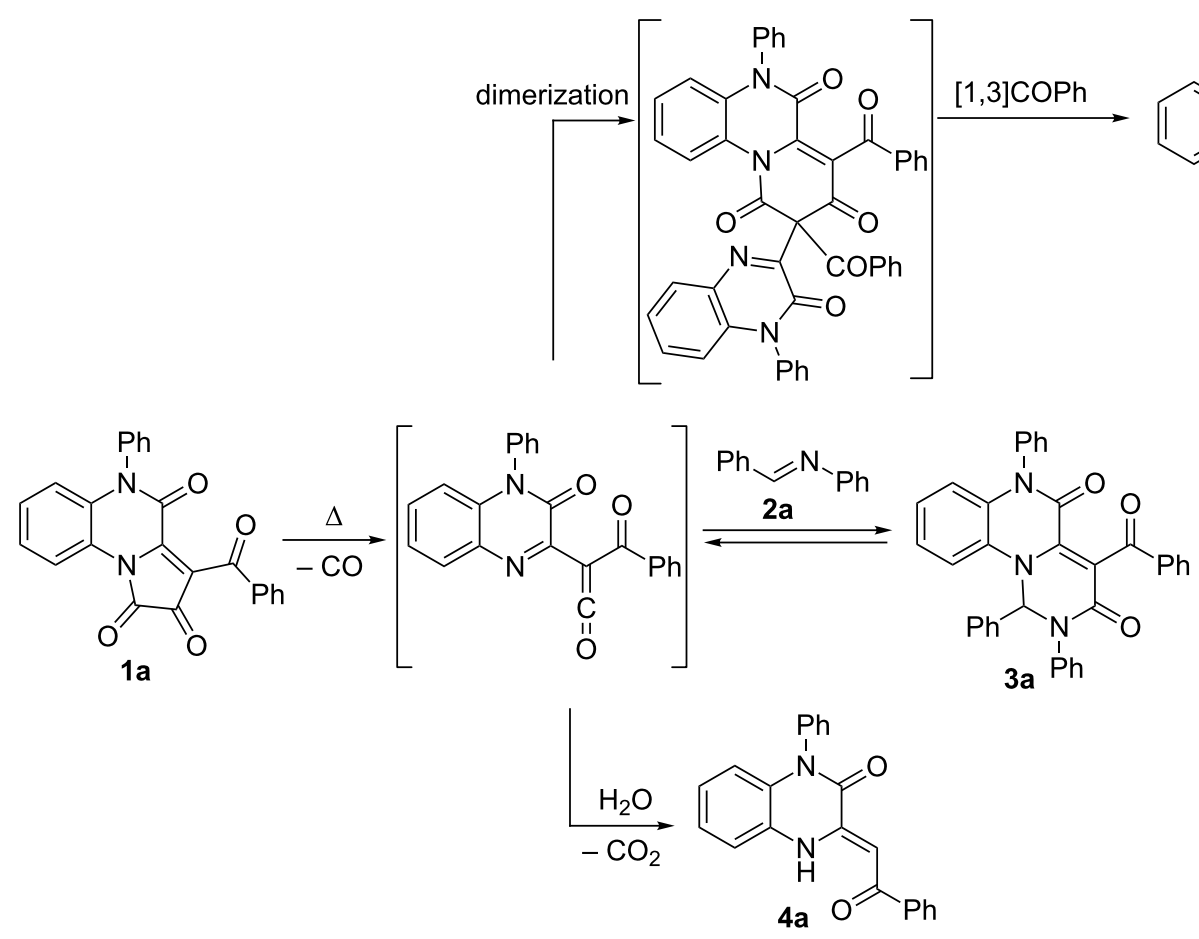

Scheme 2: Side-reactions concurring with intermolecular trapping of ketene generated from PQT 1a by benzalaniline (2a). 
$\mathrm{R}^{4}=\mathrm{N}_{\mathrm{R}^{3}}$

2a, $\mathrm{R}^{3}, \mathrm{R}^{4}=\mathrm{Ph}$

(1)

2b, $\mathrm{R}^{3}=\mathrm{Bn}, \mathrm{R}^{4}=\mathrm{C}_{6} \mathrm{H}_{4} \mathrm{Br}-4$

2c, $\mathrm{R}^{3}=\mathrm{C}_{6} \mathrm{H}_{4} \mathrm{OMe}-4, \mathrm{R}^{4}=\mathrm{C}_{6} \mathrm{H}_{4} \mathrm{Br}-4$

2d, $\mathrm{R}^{3}=\mathrm{C}_{6} \mathrm{H}_{4} \mathrm{Cl}-4, \mathrm{R}^{4}=\mathrm{C}_{6} \mathrm{H}_{3}(\mathrm{OMe})_{2}-3,4$

solvent free,

2-3 min,

onset decarbonylation temperature of 1

1a, $R^{1}, R^{2}=P h$

1b, $\mathrm{R}^{1}=\mathrm{Ph}, \mathrm{R}^{2}=\mathrm{C}_{6} \mathrm{H}_{4} \mathrm{Cl}-4$

1c, $\mathrm{R}^{1}=\mathrm{Ph}, \mathrm{R}^{2}=\mathrm{C}_{6} \mathrm{H}_{4} \mathrm{OMe}-4$

1d, $\mathrm{R}^{1}=\mathrm{Ph}, \mathrm{R}^{2}=\mathrm{C}_{6} \mathrm{H}_{4} \mathrm{NO}_{2}-4$

1e, $R^{1}=P h, R^{2}=O E t$

1f, $R^{1}=P h, R^{2}=O M e$

1g, $R^{1}=H, R^{2}=P h$

1h, $R^{1}=H, R^{2}=O E t$<smiles>O=C(c1ccccc1)c1c(-c2ccccc2)c(-c2ccccc2)c(=O)n2c1C(=O)N(c1ccccc1)C2c1ccccc1</smiles>

3a (85\%)<smiles>COc1ccc(C(=O)C2=C3C(=O)N(c4ccccc4)c4ccccc4N3C(c3ccc(Br)cc3)N(Cc3ccccc3)C2=O)cc1</smiles>

3e $(79 \%)$<smiles>COc1ccc(N2C(=O)C(C(=O)c3ccc(Cl)cc3)=C3C(=O)N(c4ccccc4)c4ccccc4N3C2c2ccc(Br)cc2)cc1</smiles>

$3 i$ (82\%)<smiles>COC(=O)C1=C2C(=O)N(c3ccccc3)c3ccccc3N2C(c2ccc(Br)cc2)N(c2ccc(OC)cc2)C1=O</smiles>

$3 \mathrm{~m}(84 \%)$<smiles>O=C(C1=C2C(=O)N(c3ccccc3)c3ccccc3N2C(c2ccc(Br)cc2)N(Cc2ccccc2)C1=O)c1ccccc1</smiles>

3b (86\%)<smiles>COc1ccc(C(=O)C2=C3C(=O)N(c4ccccc4)c4ccccc4N3C(c3ccccc3)N(c3ccccc3)C2=O)cc1</smiles>

$3 c(81 \%)$

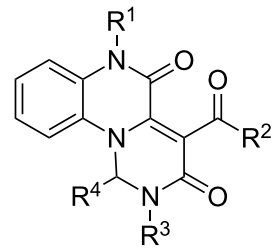

3a-n<smiles></smiles>

3d $(87 \%)$<smiles>O=C(C1=C2C(=O)N(c3ccccc3)c3ccccc3N2C(c2ccccc2)N(c2ccccc2)C1=O)c1ccc([N+](=O)[O-])cc1</smiles><smiles>O=C(C1=C2C(=O)N(c3ccccc3)c3ccccc3N2C(c2ccc(Br)cc2)N(Cc2ccccc2)C1=O)c1ccc([N+](=O)[O-])cc1</smiles><smiles>CCOC(=O)C1=C2C(=O)N(c3ccccc3)c3ccccc3N2C(c2ccccc2)N(c2ccccc2)C1=O</smiles>

$3 \mathbf{f}(83 \%)$

$3 g(80 \%)$

3h $(87 \%)$<smiles>CCOC(=O)C1=C2C(=O)N(c3ccccc3)c3ccccc3N2C(c2ccc(Br)cc2)N(c2ccc(OC)cc2)C1=O</smiles>

3j $(84 \%)$<smiles>CCOC(=O)C1=C2C(=O)N(c3ccccc3)c3ccccc3N2C(c2ccc(Br)cc2)N(Cc2ccccc2)C1=O</smiles><smiles>COC(=O)C1=C2C(=O)N(c3ccccc3)c3ccccc3N2C(c2ccccc2)N(c2ccccc2)C1=O</smiles>

3k $(79 \%)$

3I (86\%)<smiles>COC(=O)C1=C2C(=O)N(c3ccccc3)c3ccccc3N2C(c2ccc(OC)c(OC)c2)C2N(c3ccccc3)C(=O)C1N2c1ccc(Cl)cc1</smiles><smiles>CCOC(=O)C1=C2C(=O)Nc3ccccc3N2C(c2ccccc2)N(c2ccccc2)C1=O</smiles><smiles></smiles>

$30(0 \%)$

$3 p(0 \%)$

Figure 5: Scope of the intermolecular trapping of ketenes generated from PQTs $\mathbf{1 a}-\mathbf{h}$ by Schiff bases $\mathbf{2 a - d}$ under solvent-free conditions. 


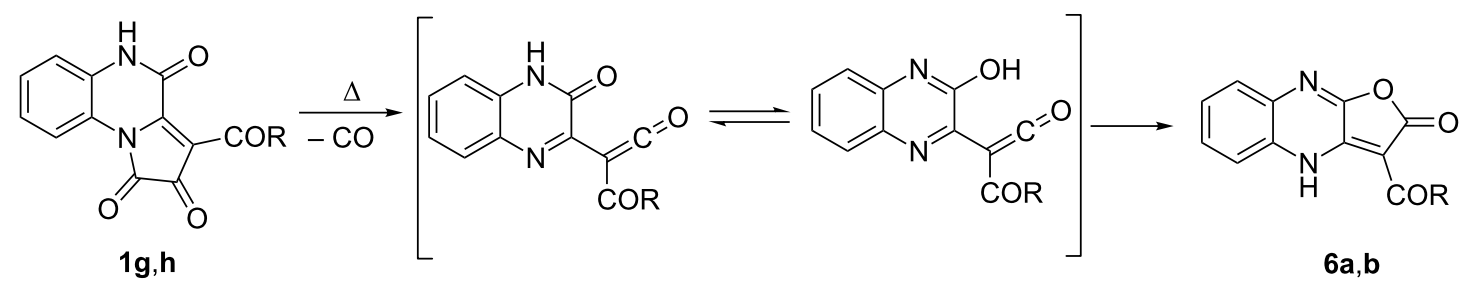

Scheme 3: Formation of furoquinoxalines $6 \mathbf{a}, \mathbf{b}$ via intramolecular cyclization in ketenes generated from PQTs $1 \mathrm{~g}, \mathbf{h}$

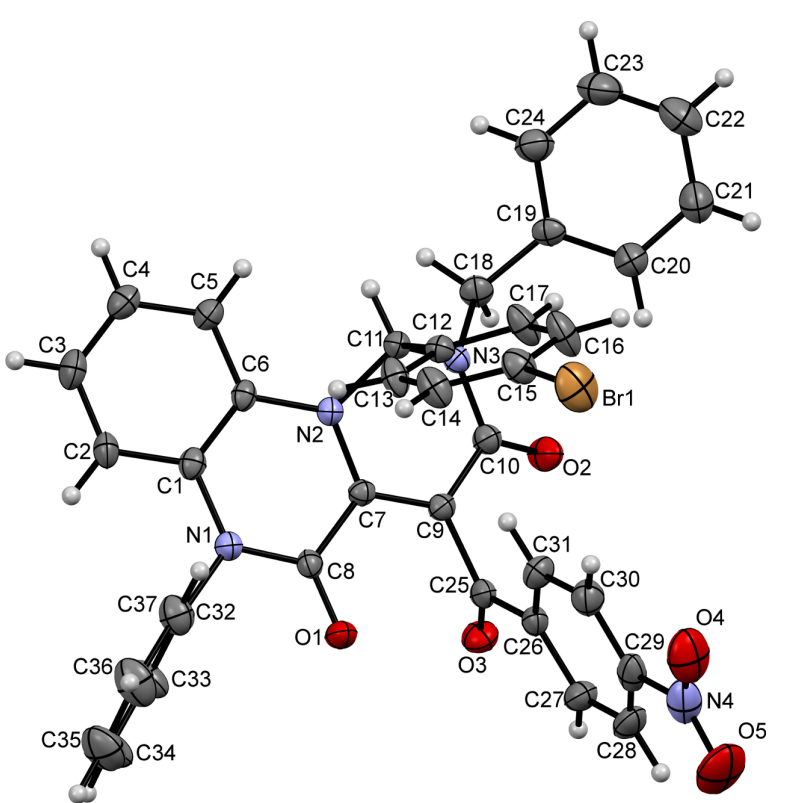

Figure 6: ORTEP drawing of compound $\mathbf{3 g}$ (CCDC 1834011) showing thermal ellipsoids at the $30 \%$ probability level.

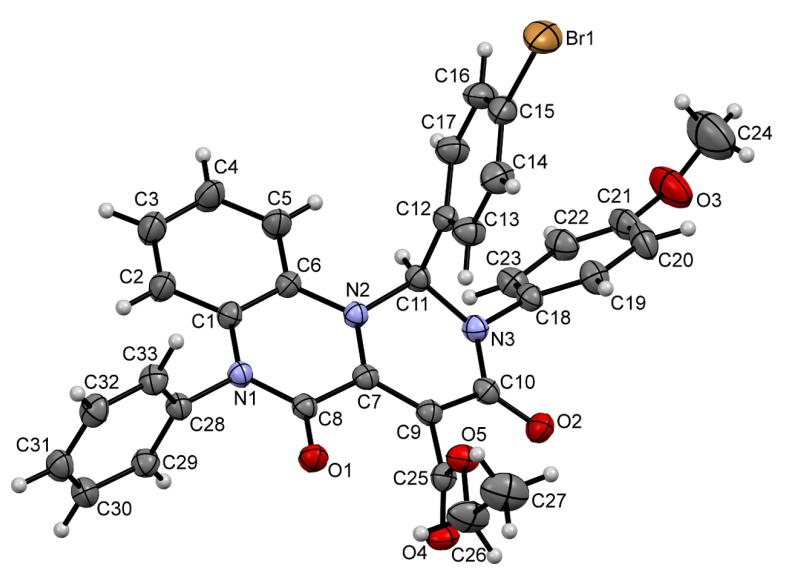

Figure 7: ORTEP drawing of compound 3j (CCDC 1834012) showing thermal ellipsoids at the $30 \%$ probability level.

\section{Conclusion}

We have developed a facile synthesis of pyrimido[1,6a]quinoxaline derivatives via the intermolecular trapping of thermally generated acyl(quinoxalin-2-yl)ketenes by Schiff bases. The reaction proceeds under solvent-free conditions without any additives and catalysts. The elaborated method might be applicable to the syntheses of pharmaceutically important substances.

\section{Supporting Information}

\section{Supporting Information File 1}

Experimental details, copies of ${ }^{1} \mathrm{H}$ and ${ }^{13} \mathrm{C}$ NMR spectra of pyrimido[1,6- $a$ ] quinoxalines 3a-n, STA plots of PQT 1a-h and X-ray crystal structure details of compounds $\mathbf{3 g}, \mathbf{j}$. [https://www.beilstein-journals.org/bjoc/content/ supplementary/1860-5397-14-147-S1.pdf]

\section{Acknowledgements}

This work was supported by the Russian Science Foundation, project \# 17-73-10210.

\section{ORCID ${ }^{\circledR}$ iDs}

Svetlana O. Kasatkina - https://orcid.org/0000-0002-7943-7525 Ekaterina E. Stepanova - https://orcid.org/0000-0002-5851-3082 Andrey N. Maslivets - https://orcid.org/0000-0001-7148-4450

\section{References}

1. Pereira, J. A.; Pessoa, A. M.; Cordeiro, M. N. D. S.; Fernandes, R.; Prudêncio, C.; Noronha, J. P.; Vieira, M. Eur. J. Med. Chem. 2015, 97, 664-672. doi:10.1016/j.ejmech.2014.06.058

2. Tariq, S.; Somakala, K.; Amir, M. Eur. J. Med. Chem. 2018, 143, 542-557. doi:10.1016/j.ejmech.2017.11.064

3. Miyashiro, J.; Woods, K. W.; Park, C. H.; Liu, X.; Shi, Y.; Johnson, E. F.; Bouska, J. J.; Olson, A. M.; Luo, Y.; Fry, E. H.; Giranda, V. L.; Penning, T. D. Bioorg. Med. Chem. Lett. 2009, 19, 4050-4054. doi:10.1016/j.bmcl.2009.06.016

4. Liu, R.; Huang, Z.; Murray, M. G.; Guo, X.; Liu, G. J. Med. Chem. 2011, 54, 5747-5768. doi:10.1021/jm200394x 
5. Sabb, A. L.; Welmaker, G. S.; Nelson, J. A. 2,3,4,4a-Tetrahydro-1H-pyrazino(1,2-a)quinoxalin-5(6H)one derivates being $5 \mathrm{HT}_{2 \mathrm{C}}$ agonists. WO Patent WO0035922 A1, June 6, 2000.

6. Rosenzweig-Lipson, S.; Zhang, J.; Mazandarani, H.; Harrison, B. L.; Sabb, A.; Sabalski, J.; Stack, G.; Welmaker, G.; Barrett, J. E.; Dunlop, J. Brain Res. 2006, 1073-1074, 240-251. doi:10.1016/j.brainres.2005.12.052

7. Hayes, D. J.; Mosher, T. M.; Greenshaw, A. J. Behav. Brain Res. 2009, 197, 323-330. doi:10.1016/j.bbr.2008.08.034

8. May, J. A.; Zinke, P. W. (R)-8,9-Dichloro-2,3,4,4a-tetrahydro-1H,6H-pyrazino[1,2-a]quinoxalin-5 -one for controlling IOP and treating glaucoma. U.S. Patent US2006211700 A1, Sept 21, 2006.

9. Kushnir, O. V.; Vovk, M. V. Russ. J. Org. Chem. 2010, 46, 890-893. doi:10.1134/s1070428010060187

10. Preciado, S.; Vicente-García, E.; Llabrés, S.; Luque, F. J.; Lavilla, R. Angew. Chem. 2012, 124, 6980-6983. doi:10.1002/ange.201202927

11. Sawant, R. T.; Stevens, M. Y.; Sköld, C.; Odell, L. R. Org. Lett. 2016, 18, 5392-5395. doi:10.1021/acs.orglett.6b02774

12. Kappe, T.; Linnau, Y.; Stadlbauer, W. Monatsh. Chem. 1977, 108, 103-111. doi:10.1007/BF00900912

13. Ames, D. E.; Brohi, M. I. J. Chem. Soc., Perkin Trans. 11980 , 1384-1389. doi:10.1039/p19800001384

14. Adegoke, E. A.; Alo, B. J. Heterocycl. Chem. 1983, 20, 1509-1512. doi:10.1002/jhet.5570200614

15. Chernavskaya, L. N.; Kholodova, N. V.; Blagorodov, S. G.; Dmitrieva, N. A. Pharm. Chem. J. 1984, 18, 413-416. doi:10.1007/BF00776797

16. Kurasawa, Y.; Nemoto, Y.; Sakakura, A.; Ogura, M.; Takada, A. Chem. Pharm. Bull. 1984, 32, 3366-3372. doi:10.1248/cpb.32.3366

17. Kawahara, N.; Shimamori, T.; Itoh, T.; Takayanagi, H.; Ogura, H. Chem. Pharm. Bull. 1987, 35, 457-467. doi:10.1248/cpb.35.457

18. Kawahara, N.; Shimamori, T.; Itoh, T.; Takayanagi, H.; Ogura, H. J. Heterocycl. Chem. 1989, 26, 847-852. doi:10.1002/jhet.5570260362

19. Öcal, N.; Turgut, Z.; Kaban, Ş. J. Heterocycl. Chem. 1998, 35 , 1349-1351. doi:10.1002/jhet.5570350620

20. Maslivets, A. N.; Golovnina, O. V.; Krasnykh, O. P.; Aliev, Z. G. Chem. Heterocycl. Compd. 2000, 36, 615-616. doi:10.1007/bf02290858

21. Lisovenko, N. Y.; Krasnykh, O. P.; Aliev, Z. G.; Vostrov, E. S.; Tarasova, O. P.; Maslivets, A. N. Chem. Heterocycl. Compd. 2001, 37, 1314-1316. doi:10.1023/A:1013886602711

22. Duffy, K. J.; Haltiwanger, R. C.; Freyer, A. J.; Li, F.; Luengo, J. I.; Cheng, H.-Y. J. Chem. Soc., Perkin Trans. 2 2002, 181-185. doi:10.1039/b102755g

23. Maslivets, A. N.; Bozdyreva, K. S.; Smirnova, I. V.; Tolmacheva, I. A.; Mashevskaya, I. V. Chem. Heterocycl. Compd. 2002, 38, 498-499. doi:10.1023/A:1016056011167

24. Maslivets, A. N.; Lisovenko, N. Y.; Krasnykh, O. P.; Tarasova, O. P.; Aliev, Z. G.; Atovmyan, L. O. Russ. Chem. Bull. 2002, 51, 850-853. doi:10.1023/A:1016097120253

25. García, M. B.; Orelli, L. R.; Magri, M. L.; Perillo, I. A. Synthesis 2002, 2687-2690. doi:10.1055/s-2002-35980

26. Bunce, R. A.; Herron, D. M.; Hale, L. Y. J. Heterocycl. Chem. 2003, 40, 1031-1039. doi:10.1002/jhet.5570400611

27. Chicharro, R.; de Castro, S.; Reino, J. L.; Arán, V. J. Eur. J. Org. Chem. 2003, 2314-2326. doi:10.1002/ejoc.200300028

28. Maslivets, A. N.; Aliev, Z. G.; Krasnykh, O. P.; Golovnina, O. V.; Atovmyan, L. O. Chem. Heterocycl. Compd. 2004, 40, 1295-1299. doi:10.1007/s10593-005-0060-4
29. Bozdyreva, K. S.; Smirnova, I. V.; Maslivets, A. N. Russ. J. Org. Chem. 2005, 41, 1081-1088. doi:10.1007/s11178-005-0296-6

30. Ma, Y.; Luo, W.; Camplo, M.; Liu, Z.; Hider, R. C. Bioorg. Med. Chem. Lett. 2005, 15, 3450-3452. doi:10.1016/j.bmcl.2005.05.010

31. Tanimori, S.; Nishimura, T.; Kirihata, M. Bioorg. Med. Chem. Lett. 2009, 19, 4119-4121. doi:10.1016/j.bmcl.2009.06.007

32. Yavari, I.; Souri, S.; Sirouspour, M.; Bayat, M. J. Synlett 2009, 1921-1922. doi:10.1055/s-0029-1217542

33. Luo, X.; Chenard, E.; Martens, P.; Cheng, Y.-X.; Tomaszewski, M. J. Org. Lett. 2010, 12, 3574-3577. doi:10.1021/ol101454x

34. Xu, L.; Jiang, Y.; Ma, D. Synlett 2010, 2285-2288. doi:10.1055/s-0030-1258030

35. Tanimori, S.; Kashiwagi, H.; Nishimura, T.; Kirihata, M. Adv. Synth. Catal. 2010, 352, 2531-2537. doi:10.1002/adsc.201000323

36. Gulevskaya, A. V.; Nguyen, H. T. L.; Tyaglivy, A. S.; Pozharskii, A. F. Tetrahedron 2012, 68, 488-498. doi:10.1016/j.tet.2011.11.018

37. Xu, Z.; De Moliner, F.; Cappelli, A. P.; Hulme, C. Org. Lett. 2013, 15, 2738-2741. doi:10.1021/ol401068u

38. Lisovenko, N. Y.; Yukova, Y. V.; Makhmudov, R. R. Pharm. Chem. J. 2014, 47, 593-595. doi:10.1007/s11094-014-1014-x

39. Nguyen, H. T. L.; Gulevskaya, A. V.; Pozharskii, A. F.; Nelina-Nemtseva, J. I. Tetrahedron 2014, 70, 4617-4625. doi:10.1016/j.tet.2014.05.023

40. Obydennov, D. L.; Sosnovskikh, V. Y. Chem. Heterocycl. Compd. 2014, 50, 579-582. doi:10.1007/s10593-014-1510-7

41. Tanimori, S.; Inaba, U.; Kato, Y.; Ura, H.; Kashiwagi, H.; Nishimura, T.; Kirihata, M. Res. Chem. Intermed. 2014, 40, 2157-2164. doi:10.1007/s11164-014-1593-x

42. Miyamaru, S.; Umezu, K.; Ito, A.; Shimizu, M. Eur. J. Org. Chem. 2015, 3327-3337. doi:10.1002/ejoc.201500225

43. Rezvanian, A. Tetrahedron 2016, 72, 6428-6435. doi:10.1016/j.tet.2016.08.049

44. Azev, Y. A.; Kodess, M. I.; Ezhikova, M. A.; Ermakova, O. S.; Berseneva, V. S.; Bakulev, V. A. Mendeleev Commun. 2017, 27, 97-98. doi:10.1016/j.mencom.2017.01.032

45. Azev, Y. A.; Ermakova, O. S.; Berseneva, V. S.; Kodess, M. I.; Ezhikova, M. A.; Ganebnykh, I. N. Mendeleev Commun. 2017, 27, 637-639. doi:10.1016/j.mencom.2017.11.034

46. Soozani, A.; Keivanloo, A.; Bakherad, M. Tetrahedron 2018, 74, 150-156. doi:10.1016/j.tet.2017.11.055

47. Reber, K. P.; Tilley, S. D.; Sorensen, E. J. Chem. Soc. Rev. 2009, 38, 3022-3034. doi:10.1039/B912599J

48. Presset, M.; Coquerel, Y.; Rodriguez, J. Org. Lett. 2009, 11, 5706-5709. doi:10.1021/ol9024056

49. Presset, M.; Coquerel, Y.; Rodriguez, J. Org. Lett. 2010, 12 , 4212-4215. doi:10.1021/ol101938r

50. Leber, S.; Kollenz, G.; Wentrup, C. Beilstein J. Org. Chem. 2012, 8, 738-743. doi:10.3762/bjoc.8.83

51. Galvez, J.; Castillo, J.-C.; Quiroga, J.; Rajzmann, M.; Rodriguez, J.; Coquerel, Y. Org. Lett. 2014, 16, 4126-4129. doi:10.1021/ol5018245

52. Khlebnikov, A. F.; Novikov, M. S.; Pakalnis, V. V.; lakovenko, R. O.; Yufit, D. S. Beilstein J. Org. Chem. 2014, 10, 784-793. doi:10.3762/bjoc. 10.74

53. Cookson, R.; Barrett, T. N.; Barrett, A. G. M. Acc. Chem. Res. 2015, 48, 628-642. doi:10.1021/ar5004169

54. Kollenz, G.; Wentrup, C. Beilstein J. Org. Chem. 2018, 14, 1-10. doi:10.3762/bjoc. 14.1 
55. Silaichev, P. S.; Maslivets, A. N. Russ. J. Org. Chem. 2012, 48, 1261-1262. doi:10.1134/S1070428012090229

56. Mashevskaya, I. V.; Mokrushin, I. G.; Bozdyreva, K. S.; Maslivets, A. N. Russ. J. Org. Chem. 2011, 47, 253-257. doi:10.1134/S1070428011020151

57. Lisovenko, N. Y.; Maslivets, A. N.; Aliev, Z. G. Chem. Heterocycl. Compd. 2003, 39, 132-134. doi:10.1023/A:1023097414711

58. Lisovenko, N. Y.; Maslivets, A. N. Chem. Heterocycl. Compd. 2004, 40 247-248. doi:10.1023/B:COHC.0000027901.78882.86

59. Lisovenko, N. Y.; Maslivets, A. N.; Aliev, Z. G. Russ. J. Org. Chem. 2004, 40, 1053-1057. doi:10.1023/B:RUJO.0000045203.69639.83

60. Lisovenko, N. Y.; Maslivets, A. N.; Aliev, Z. G. Russ. J. Org. Chem. 2007, 43, 117-120. doi:10.1134/s1070428007010150

61. Nekrasov, D. D.; Obukhova, A. S.; Lisovenko, N. Y.; Roubtsov, A. E. Chem. Heterocycl. Compd. 2010, 46, 413-418. doi:10.1007/s10593-010-0525-y

62. Lisovenko, N. Y.; Nekrasov, D. D.; Karmanov, V. I. Chem. Heterocycl. Compd. 2012, 48, 1357-1360. doi:10.1007/s10593-012-1144-6

63. Masilvets, A. N.; Golovnina, O. V.; Krasnykh, O. P.; Aliev, Z. G. Chem. Heterocycl. Compd. 2000, 36, 355-356. doi:10.1007/BF02256878

64. Aliev, Z. G.; Maslivets, A. N.; Golovnina, O. V.; Krasnykh, O. P.; Atovmyan, L. O. Russ. Chem. Bull. 2001, 50, 1317-1319. doi:10.1023/A:1014091731016

\section{License and Terms}

This is an Open Access article under the terms of the Creative Commons Attribution License (http://creativecommons.org/licenses/by/4.0), which permits unrestricted use, distribution, and reproduction in any medium, provided the original work is properly cited.

The license is subject to the Beilstein Journal of Organic Chemistry terms and conditions:

(https://www.beilstein-journals.org/bjoc)

The definitive version of this article is the electronic one which can be found at:

$\underline{\text { doi:10.3762/bjoc. } 14.147}$ 